\title{
Uncertainty Model For Quantitative Precipitation Estimation Using Weather Radars
}

\author{
A. Ernesto Gómez Vargas, B. Nelson Obregón Neira, C. Lindsay Álvarez Pomar \\ Universidad Distrital Francisco José de Caldas, Bogotá D.C., Colombia
}

\begin{abstract}
This paper introduces an uncertainty model for the quantitatively estimate precipitation using weather radars. The model considers various key aspects associated to radar calibration, attenuation, and the tradeoff between accuracy and radar coverage. An S-band-radar case study is presented to illustrate particular fractional-uncertainty calculations obtained to adjust various typical radar-calibration elements such as antenna, transmitter, receiver, and some other general elements included in the radar equation. This paper is based in "Guide to the expression of Uncertainty in measurement" [1] and the results show that the fractional uncertainty calculated by the model was $40 \%$ for the reflectivity and $30 \%$ for the precipitation using the Marshall Palmer Z-R relationship.
\end{abstract}

Keywords - Quantitative Precipitation Estimation, Radar Measurements, Uncertainty, Weather Radar.

\section{INTRODUCTION}

$\mathrm{M}$ OST methods to quantitatively estimate precipitation often fail to provide information about the type of uncertainty associated to the corresponding measurement technique; therefore it seems compelling to quantify the technique-associated uncertainty together with the actual estimation results [2].

The general purpose of measuring is to determine the actual value of a particular magnitude (i.e. the variable), which in our case corresponds to precipitation. According to [3], measurement uncertainty is a way to express the idea whereby for a magnitude and its given measurement there is no unique value but an infinite number of values scattered in the vicinity of the suggested result; additionally, these values are consistent with all observations, data and knowledge gathered from the physical world, and so can be attributed to the measured magnitudes with different degrees of reliability.

Just like other measurement instruments, weather radars rely on indirect measurements of actual precipitation; that is, radar receivers use antennas to perceive information associated to hydrometeors-echo power. This information is subsequently transformed using the radar equation (for radar reflectivity) and then, a whole data interpretation process begins in order to finally yield a quantitative precipitation estimation by means of a given algorithm (or method). From this point onwards, there are a considerable number of aspects that affect radar measurements and so influence data; therefore such aspects have a great impact on the final result (i.e. precipitation levels). These aspects include the radar equation itself, calibration issues associated to the observation system (antenna, receiver and transmitter), space-time variability of the measurements and precipitation's own micro-physics, which cause quantitative precipitation estimations to have considerable uncertainty associated to its measuring process.

This paper provides a comprehensive uncertainty analysis intended for radar measurement procedures. A particular case study is analyzed in order to show quantitative results on the amount of uncertainty in the measurement system (the extent of such uncertainty). Section 2 of the paper illustrates the methodology applied to obtain the uncertainty model and section 3 shows specific case-study results. Conclusions are drawn in the final part of the paper.

\section{Methodology}

The methodology applied in this work involves various stages. There is an initial specification of the variable to be measured (i.e. precipitation), which is a function of other variables that are previously defined in the radar equation. Subsequently, the uncertainty sources associated to this process are identified e.g. anomalous propagation, attenuation, ground echo, beam partial filling, resolution, beam height, calibration, type of rain, DSD variety, evaporation and condensation [4].

\section{A. Conceptual Model}

Once the uncertainty sources were identified, a sub-set of uncertainty sources was selected, namely those related to radar calibration (i.e. antenna, transmitter and receiver), attenuation and the distance-sensitive radar resolution loss. Fig. 1 shows the conceptual map associated to the model:

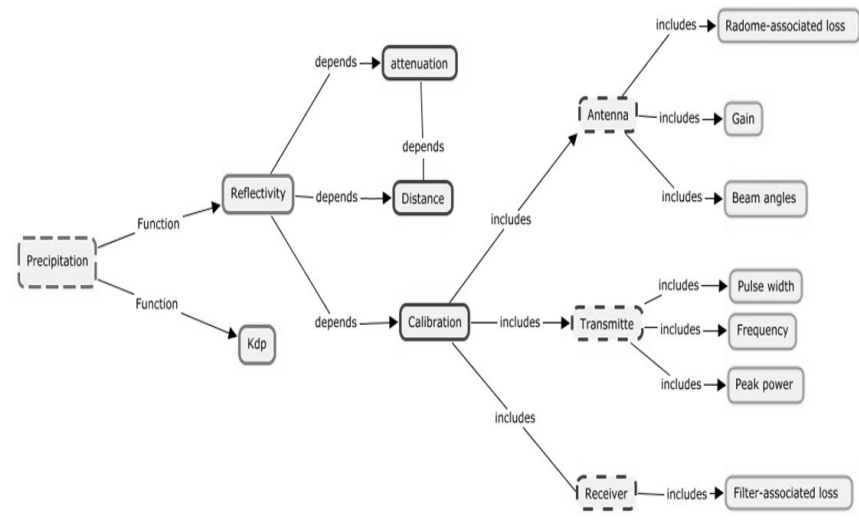

Fig, 1. Conceptual Model

\section{B. Mathematical Model}

The mathematical uncertainty-measuring model was taken from "Guide to the expression of Uncertainty in measurement". The following is a description of the model [1]:

In most cases the value of variable $\mathrm{Y}$ (precipitation) is not measured directly; instead, such a value results from measuring other $\mathrm{N}$ magnitudes, namely $X_{1}, X_{2}, X_{3}, \ldots . X_{N}$, using a functional relationship as follows:

$$
Y=f\left(X_{1}, X_{2}, X_{3}, \ldots . X_{N}\right)
$$


In some cases, the best estimation "y" of variable "Y" can be obtained from gathering " $n$ " observations, namely:

$$
y=\bar{Y}=\frac{1}{n} \sum_{k=1}^{n} Y_{k}=\frac{1}{n} \sum_{k=1}^{n} f\left(X_{1, k}, X_{2, k}, X_{3, k}, \ldots . X_{N, k}\right)
$$

The type of uncertainty associated to output estimation " $y$ " is referred to as combined typical uncertainty and it is denoted by $u_{c}$ .This particular uncertainty can be determined from the estimated typical deviation associated to each input estimate $x_{i}$, referred to as typical uncertainty and denoted by $\mathrm{u}\left(x_{i}\right)$.

Each of the input estimations $x_{i}$ as well as their corresponding uncertainty $\mathrm{u}\left(x_{i}\right)$ is obtained from a distribution of possible values of each input magnitude $x_{i}$. This probability distribution might be based on a series of " $n$ " observations $x_{i, k}$ taken from the set of observations $x_{i}$ (type A), or else, the distribution may be assumed (type B).

The type-A uncertainty assessment is obtained from various observations and it is calculated using the following expression:

$$
u(x)=\frac{\sqrt{\sum_{j=1}^{n}\left(x_{j}-\bar{x}\right)^{2}}}{n(n-1)}
$$

Where:

$$
\bar{x}=\frac{1}{n} \sum_{j=1}^{n} x_{j}
$$

For a single estimation $\mathrm{X}_{\mathrm{i}}$ associated to an input magnitude $\mathrm{X}_{\mathrm{i}}$ that was not obtained from repeated observations, the corresponding associated estimated variance, namely $\mathrm{u}^{2}\left(\mathrm{x}_{\mathrm{i}}\right)$, or the typical uncertainty $\mathrm{u}\left(\mathrm{x}_{\mathrm{i}}\right)$, can be established through scientific decision that is based on all the available information about the possible variability of $X_{i}$. The sort of information gathered may include:

- Results from previous measurements.

- Experience or general knowledge about the behavior and the properties of the specific materials and instruments.

- Manufacturing specifications (from suppliers).

- Data provided by certified calibrations or other types of certified processes

- Uncertainty previously assigned to reference values, which may be taken from books and manuals.

The values of $u^{2}\left(x_{i}\right)$ and $u\left(x_{i}\right)$ that are assessed as stated are called Type-B variance and Type-B typical uncertainty, respectively.

The uncertainty of $x_{i}$ is not always expressed as a multiple of a typical deviation. Instead, it is possible to define a specific interval that corresponds to a particular reliability level (e.g. $90 \%, 95 \%$ or 99\%). Unless stated otherwise, it can be assumed that a normal distribution has been used to calculate uncertainty; this yields the typical uncertainty of $x_{i}$ simply by dividing the given uncertainty value by the corresponding factor in the normal distribution. Such a factor for the three aforementioned reliability levels is 1.64, 1.96 and 2.58 , respectively.

In order to obtain the combined typical uncertainty estimation, namely $\mathrm{u}_{\mathrm{c}}(\mathrm{y})$, in the case where all input magnitudes are independent, we take the positive square root of combined variance $u_{c}^{2}(y)$, given by:

$$
u_{c}^{2}(y)=\sum_{i=1}^{N}\left[\frac{\partial f}{\partial x_{i}}\right]^{2} u^{2}\left(x_{i}\right)
$$

Where $f$ is the function that defines the variable itself as a function of variables $X_{i}$.

Each $\mathrm{u}\left(x_{i}\right)$ represents a single typical uncertainty that is assessed as previously described (either Type-A assessment or Type-B assessment). The combined typical uncertainty $\mathrm{u}_{\mathrm{c}}(\mathrm{y})$ is a typical deviation that is estimated and characterized according to the dispersion of the potential reasonable values that can be attributed to variable "Y".

Partial derivatives (i.e. $\partial \mathrm{f} / \partial \mathrm{xi}$ ), also known as sensitivity coefficients, describe the variation of output estimation " $y$ " as a function of the variations in the input estimation values $x_{1}, x_{2}, x_{3}, \ldots x_{N}$.

It is worth mentioning that the previous equation holds only if the input magnitudes $x_{i}$ are independent or uncorrelated. In case some of the values of $x_{i}$ are highly correlated, it is essential to consider such correlations as follows:

$$
\begin{aligned}
u_{c}^{2}(y)=\sum_{i=1}^{N} \sum_{j=1}^{N} \frac{\partial f}{\partial x_{i}} \frac{\partial f}{\partial x_{j}} u\left(x_{i}, x_{j}\right) & \\
& =\sum_{i=1}^{N}\left[\frac{\partial f}{\partial x_{i}}\right]^{2} u^{2}\left(x_{i}\right) \\
& +2 \sum_{i=1}^{N-1} \sum_{j=i+1}^{N} \frac{\partial f}{\partial x_{i}} \frac{\partial f}{\partial x_{j}} u\left(x_{i}, x_{j}\right)
\end{aligned}
$$

Where $x_{i}$ and $x_{j}$ correspond to the estimations of $\mathrm{X}_{\mathrm{i}}$ and $\mathrm{X}_{\mathrm{j}}$; and $\mathrm{u}\left(x_{i}, x_{j}\right)=\mathrm{u}\left(x_{j}, x_{i}\right)$ represents the estimated covariance associated to $x_{i}$ and $x_{j}$. The extent of the correlation between $x_{i}$ and $x_{j}$ is determined by the correlation coefficient as follows:

$$
r\left(x_{i}, x_{j}\right)=\frac{u\left(x_{i}, x_{j}\right)}{u\left(x_{i}\right) \mathrm{u}\left(x_{j}\right)}
$$

Where $r\left(x_{i}, x_{j}\right)=r\left(x_{j}, x_{i}\right)$ and $-1 \leq r\left(x_{i}, x_{j}\right) \leq+1$. If estimations $x_{i}$ and $x_{j}$ are independent, $r\left(x_{i}, x_{j}\right)=0$, and so a variation in one of the estimation values does not imply a variation in the other value.

Fractional uncertainty is defined as the ratio of typical uncertainty to the value of the best magnitude estimate, namely:

$$
\text { Fractional Uncertainty }=\frac{u\left(x_{i}\right)}{x_{i}}
$$

Although $u_{c}(\mathrm{y})$ can be generally employed as an expression of uncertainty for a particular measurement result, it is often necessary (in certain commercial, industrial or regulatory applications as well as in the fields of health and security) to provide uncertainty measurements that clearly define an interval in which most of the distribution (reasonably attributed values) of the variable are expected to fall.

The new expression for uncertainty, which satisfies the intervaldefinition requirement, is referred to as expanded uncertainty and is denoted by $U$. Expanded uncertainty (U) is obtained by multiplying the combined typical uncertainty $u_{c}(\mathrm{y})$ by a given coverage factor $(\mathrm{k})$ : 


$$
U=k u_{c}(y)
$$

In general, $\mathrm{k}$ takes values between 2 and 3. Experience and broad knowledge about the way measurement results should be handled may facilitate choosing a convenient value for $\mathrm{k}$.

\section{Uncertainty due to calibrations involving radar, distance and attenuation}

Taking the radar equation defined by Probert Jones [5] as a starting point, a standard uncertainty calculation can be stated as follows:

$$
P_{r}=\frac{\pi^{3} P_{t x} G^{2} \theta \phi h|K|^{2} Z}{1024 \ln (2) \lambda^{2} r^{2}}
$$

Where : $\mathrm{P}_{\mathrm{r}}$ : received power at the radar

$$
\begin{aligned}
& \mathrm{P}_{\mathrm{tx}} \text { : radiated power from the transmitter } \\
& \lambda: \text { wavelength }(\mathrm{c} / \mathrm{f}) \\
& \mathrm{Z}: \text { Radar-reflectivity factor } \\
& \mathrm{K}: \text { refraction complex index } \\
& \mathrm{h}: \text { spatial pulse length }(\mathrm{c} \tau) \\
& \mathrm{G} \text { : antenna gain } \\
& \mathrm{r} \text { : distance between the target and the radar's antenna } \\
& \theta, \phi: \text { beam angles of the radar }
\end{aligned}
$$

By solving for reflectivity, and also considering that $\mathrm{h}=\mathrm{c} \tau, \theta=\phi$ and $\lambda=\mathrm{c} / \mathrm{f}$, the following expression is obtained:

$$
Z=\frac{2^{10} \ln (2) c}{\pi^{3}|K|^{2} G^{2} \theta^{2} P_{t x} \tau f^{2}} P_{r} r^{2}
$$

The addition of some terms that were not included by Jones, such as propagation loss $\left(\mathrm{L}_{\mathrm{p}}\right)$, receiving filter loss $\left(\mathrm{L}_{\mathrm{MF}}\right)$ [6][7] and a radomeassociated loss $\left(\mathrm{L}_{\mathrm{RD}}\right)$, yields the following expression:

$$
Z=\frac{2^{10} \ln (2) c \quad L_{R D}^{2} \quad L_{M F}}{\pi^{3}|K|^{2} G_{A}^{2} \theta^{2} P_{t x} \tau f^{2}} L_{p}^{2} P_{r} r^{2}
$$

For radars that are in normal operation conditions, it is always possible to simplify the radar equation since most of the terms are constants; thus the following expression can be used:

$$
Z=C_{R} L_{p}^{2} P_{r} r^{2}
$$

Where $C_{R}$ is the radar constant, defined by:

$$
C_{R}=\frac{2^{10} \ln (2) c L_{R D}^{2} \quad L_{M F}}{\pi^{3}|K|^{2} G_{A}^{2} \theta^{2} P_{t x} \tau f^{2}}
$$

\begin{tabular}{|c|c|c|}
\hline Elements & $\begin{array}{c}\text { Factors to be considered in } \\
\text { calibration }\end{array}$ & $\begin{array}{c}\text { Standard } \\
\text { uncertainty }\end{array}$ \\
\hline $\begin{array}{c}\text { Radar Calibration } \\
\text { Antenna }\end{array}$ & $\begin{array}{ll}\text { - } & \text { Gain }\left(G_{A}\right) . \\
\text { - } & -3 \mathrm{~dB} \text { beam-width angles }(\theta, \phi) . \\
\text { - } & \text { Antenna constant } \mathrm{C}_{\mathrm{ANT}}=\mathrm{G}_{\mathrm{A}}^{2} \theta^{2} . \\
\text { - } & \text { Radome-associated loss }\left(\mathrm{L}_{\mathrm{RD}}\right) .\end{array}$ & $\begin{array}{l}\text { - } U\left(G_{A}\right) \\
\text { - } U(\theta, \phi) \\
\text { - } U\left(C_{A N T}\right) \\
\text { - } U\left(L_{R D}\right)\end{array}$ \\
\hline $\begin{array}{l}\text { Radar Calibration } \\
\text { Transmitter }\end{array}$ & $\begin{array}{ll}\text { - } & \text { Pulse length }(\boldsymbol{\tau}) \text {. } \\
\text { - } & \text { Frequency }(\mathrm{f}) \text { and PRF. } \\
\text { - } & \text { Peak power }\left(\mathrm{P}_{\mathrm{tx}}\right)\end{array}$ & $\begin{array}{l}\text { - } U(\tau) \\
\text { - } U(\mathrm{f}) \\
\text { - } U\left(\mathrm{P}_{\mathrm{tx}}\right) \\
\end{array}$ \\
\hline $\begin{array}{l}\text { Radar Calibration } \\
\text { Receiver: } \\
\end{array}$ & $\begin{array}{ll} & \text { Gain }(\mathrm{G}) . \\
\text { - } & \text { Filter-associated loss }\left(\mathrm{L}_{\mathrm{MF}}\right) \\
\end{array}$ & $\begin{array}{ll} & U(\mathrm{G}) \\
\text { - } & U\left(\mathrm{~L}_{\mathrm{MF}}\right) \\
\end{array}$ \\
\hline Attenuation & - Propagation loss $\left(\mathrm{L}_{\mathrm{p}}\right)$ & - $U\left(\mathrm{~L}_{\mathrm{p}}\right)$ \\
\hline Resolution Space & - Coverage range (r). & - $U(\mathrm{r})$ \\
\hline $\begin{array}{l}\text { Physical } \\
\text { constants: }\end{array}$ & $\begin{array}{l}\text { - Wave speed } \mathrm{c}=2.9978^{*} 10^{\wedge} 8 \mathrm{~m} / \mathrm{s} \text {. } \\
\text { - Dielectric constant }\left|\mathrm{K}_{\mathrm{W}}\right|^{2}=0.93 .\end{array}$ & $\begin{array}{ll} & U(\mathrm{c}) \\
\text { - } & U\left(\mathrm{~K}_{\mathrm{W}}\right) \\
\end{array}$ \\
\hline Measurements & $\begin{array}{l}\text { - } \text { Reflectivity }(\mathrm{Z}) \\
\text { - } \\
\text { Received power }\left(\mathrm{P}_{\mathrm{r}}\right) \text {. }\end{array}$ & $\begin{array}{ll}\text { - } U(\mathrm{Z}) \\
\text { - } U\left(\mathrm{P}_{\mathrm{r}}\right)\end{array}$ \\
\hline
\end{tabular}

By further elaborating on the previous expression, we obtain:

$$
C T E=\frac{2^{10} \ln (2) c}{\pi^{3}|K|^{2}}
$$

Where:

$$
C T E=\frac{2^{10} \ln (2) c}{\pi^{3}|K|^{2}} \text { y } C_{A N T}=G_{A}^{2} \theta^{2}
$$

Aspects such as antenna gain, beam angles, frequency accuracy, pulse length, and loss estimations (in radome and in the receiving filters) constitute some of the many factors that should be considered in a radar calibration process [8]. The following is a list of the most relevant factors to radar calibration:
TABLE I

FACTORS TO BE CONSIDERED IN UNCERTAINTY MODELS [8].

Regarding the various uncertainty sources that appear in the table above as independent (except for the existing correlation between the transmitting antenna gain and its $-3 \mathrm{~dB}$ beam angles, which are handled together within the antenna constant), the following general expression is thus obtained in order to determine the combined uncertainty that lies in the corresponding reflectivity $(\mathrm{Z})$ measurement:

$u_{c}^{2}(Z)=\left[\frac{\partial Z}{\partial C_{R}}\right]^{2} u^{2}\left(C_{R}\right)+\left[\frac{\partial Z}{\partial P_{r}}\right]^{2} u^{2}\left(P_{r}\right)+\left[\frac{\partial Z}{\partial L_{p}}\right]^{2} u^{2}\left(L_{p}\right)+\left[\frac{\partial Z}{\partial r}\right]^{2} u^{2}(\mathrm{r})$

Similarly, for $C_{R}$ :

$$
\begin{aligned}
& u_{c}^{2}\left(C_{R}\right)=\left[\frac{\partial C_{R}}{\partial C_{A N T}}\right]^{2} u^{2}\left(C_{A N T}\right)+\left[\frac{\partial C_{R}}{\partial L_{R D}}\right]^{2} u^{2}\left(L_{R D}\right)+\left[\frac{\partial C_{R}}{\partial \tau}\right]^{2} u^{2}(\tau) \\
& +\left[\frac{\partial C_{R}}{\partial \mathrm{f}}\right]^{2} u^{2}(\mathrm{f})+\left[\frac{\partial C_{R}}{\partial \mathrm{P}_{\mathrm{tx}}}\right]^{2} u^{2}\left(\mathrm{P}_{\mathrm{tx}}\right)+\left[\frac{\partial C_{R}}{\partial \mathrm{L}_{\mathrm{MF}}}\right]^{2} u^{2}\left(\mathrm{~L}_{\mathrm{MF}}\right)
\end{aligned}
$$

The following table shows the results in terms of partial derivatives:

TABLE II

PARTIAL DERIVATIVES FOR THE UNCERTAINTY MODEL

\begin{tabular}{cccc}
\hline & & $Z=C_{R} L_{p}^{2} P_{r} r^{2}$ & $\frac{Z}{C_{R}}$ \\
\hline$C_{1}$ & $\frac{\partial Z}{\partial C_{R}}$ & $L_{p}^{2} P_{r} r^{2}$ & $2 \frac{Z}{L_{p}}$ \\
$C_{2}$ & $\frac{\partial Z}{\partial L_{p}}$ & $2 C_{R} L_{p} P_{r} r^{2}$ & $\frac{Z}{P_{r}}$ \\
$C_{3}$ & $\frac{\partial Z}{\partial P_{r}}$ & $C_{R} L_{p}^{2} r^{2}$ & $2 \frac{Z}{r}$ \\
$C_{4}$ & $\frac{\partial Z}{\partial r}$ & $2 C_{R} L_{p}^{2} P_{r} \mathrm{r}$ & $-2 \frac{C_{R}}{C_{A N T}}$ \\
\hline$C_{A}$ & $\frac{\partial C_{R}}{\partial C_{A N T}}$ & $-2 C T E \frac{L_{R D}^{2} L_{M F}}{C_{A N T}^{2} P_{t x} \tau f^{2}}$ & $2 \frac{C_{R}}{L_{R D}}$ \\
$C_{B}$ & $\frac{\partial C_{R}}{\partial L_{R D}}$ & $2 * C T E \frac{L_{R D} L_{M F}}{C_{A N T} P_{t x} \tau f^{2}}$ & $-\frac{C_{R}}{\tau}$ \\
$C_{C}$ & $\frac{\partial C_{R}}{\partial \tau}$ & $-C T E \frac{L_{R D}^{2} L_{M F}}{C_{A N T} P_{t x} \tau^{2} f^{2}}$ & $-2 \frac{C_{R}}{f}$ \\
$C_{D}$ & $\frac{\partial C_{R}}{\partial \mathrm{f}}$ & $-2 * C T E \frac{L_{R D}^{2} L_{M F}}{C_{A N T} P_{t x} \tau f^{3}}$ & $-\frac{C_{R}}{P_{t x}}$ \\
$C_{E}$ & $\frac{\partial C_{R}}{\partial \mathrm{P}_{\mathrm{tx}}}$ & $-C T E \frac{L_{R D}^{2} L_{M F}}{C_{A N T} P_{t x}^{2} \tau f^{2}}$ & $\frac{C_{R}}{L_{M F}}$ \\
$C_{F}$ & $\frac{\partial C_{R}}{\partial \mathrm{L}_{\mathrm{MF}}}$ & $C T E \frac{L_{R D}^{2} L_{p}^{2}}{C_{A N T} P_{t x} \tau f^{2}}$ & \\
\hline
\end{tabular}


By using the partial derivatives, the combined uncertainty that occurs when measuring reflectivity $\mathrm{Z}$ can be expressed as follows:

$$
\begin{aligned}
& u_{c}^{2}(Z)=\left[\frac{Z}{C_{R}}\right]^{2} u^{2}\left(C_{R}\right)+\left[2 \frac{Z}{L_{p}}\right]^{2} u^{2}\left(L_{p}\right)+\left[\frac{Z}{P_{r}}\right]^{2} u^{2}\left(P_{r}\right)+ \\
& {\left[2 \frac{Z}{r}\right]^{2} u^{2}(\mathrm{r})}
\end{aligned}
$$

The previous expression can be rewritten as follows:

$$
\left[\frac{u_{c}(z)}{Z}\right]^{2}=\left[\frac{u\left(C_{R}\right)}{C_{R}}\right]^{2}+\left[2 \frac{u\left(L_{p}\right)}{L_{p}}\right]^{2}+\left[\frac{u\left(P_{r}\right)}{P_{r}}\right]^{2}+\left[2 \frac{u(r)}{r}\right]^{2}
$$

This expression serves to compute the fractional uncertainty associated to reflectivity measurements by assuming independence of all the variables involved. However, given the direct relation between attenuation and the radar's coverage range, and also assuming a corresponding correlation coefficient of 1 , the following is obtained:

$$
\left[\frac{u_{c}(z)}{Z}\right]^{2}=\left[\frac{u\left(C_{R}\right)}{C_{R}}\right]^{2}+\left[2 \frac{u\left(L_{p}\right)}{L_{p}}+2 \frac{u(r)}{r}\right]^{2}+\left[\frac{u\left(P_{r}\right)}{P_{r}}\right]^{2}
$$

Similarly, for the radar constant, the following is obtained:

$$
\begin{gathered}
u_{c}^{2}\left(C_{R}\right)=\left[-2 \frac{C_{R}}{C_{A N T}}\right]^{2} u^{2}\left(C_{A N T}\right)+\left[-2 \frac{C_{R}}{L_{R D}}\right]^{2} u^{2}\left(L_{R D}\right)+ \\
{\left[-\frac{C_{R}}{\tau}\right]^{2} u^{2}(\tau)+\left[-2 \frac{C_{R}}{f}\right]^{2} u^{2}(\mathrm{f})+\left[-\frac{C_{R}}{P_{t x}}\right]^{2} u^{2}\left(\mathrm{P}_{\mathrm{tx}}\right)+} \\
{\left[\frac{C_{R}}{L_{M F}}\right]^{2} u^{2}\left(\mathrm{~L}_{\mathrm{MF}}\right)} \\
{\left[\frac{u_{c}\left(C_{R}\right)}{C_{R}}\right]^{2}=\left[\frac{2 u\left(C_{A N T}\right)}{C_{A N T}}\right]^{2}+\left[\frac{2 u\left(L_{R D}\right)}{L_{R D}}\right]^{2}+\left[\frac{u(\tau)}{\tau}\right]^{2}+\left[\frac{2 u(\mathrm{f})}{f}\right]^{2}} \\
+\left[\frac{u\left(\mathrm{P}_{\mathrm{tx}}\right)}{P_{t x}}\right]^{2}+\left[\frac{u\left(\mathrm{~L}_{\mathrm{MF}}\right)}{L_{M F}}\right]^{2}
\end{gathered}
$$

The uncertainty associated to the antenna constant, which depends on the gain and also on the $-3 \mathrm{db}$ angles, is assumed as a correlated uncertainty with a correlation coefficient equal to 1 , thus the corresponding expression y computed as follows:

$$
\begin{aligned}
& C_{A N T}=G_{A}^{2} \theta^{2} \\
& {\left[\frac{u_{c}\left(C_{A N T}\right)^{2}}{C_{A N T}}\right]^{2}=\left[\frac{u\left(G_{A}\right)}{G_{A}}+\frac{u\left(G_{A}\right)^{2}}{G_{A}}\right]^{2}}
\end{aligned}
$$

Considering that the actual variable of interest is precipitation (R), instead of reflectivity (Z), and also involving the Marshall \& Palmer Z-R relation together with an algorithm that uses both reflectivity and the specific phase differential (for precipitation estimation), the following uncertainty expressions are obtained:

$$
\begin{gathered}
R=f\left(Z, K_{d p}\right) \quad o \quad R=f(Z) \\
u_{c}^{2}(R)=\left[\frac{\partial R}{\partial Z}\right]^{2} u^{2}(Z)+\left[\frac{\partial R}{\partial K_{d p}}\right]^{2} u^{2}\left(K_{d p}\right) \\
u_{c}^{2}(R)=\left[\frac{\partial R}{\partial Z}\right]^{2} u^{2}(Z)
\end{gathered}
$$

\section{CASE StUdy}

For this particular case study, technical information of radar, located in Brisbane-Australia, was considered (see Table II).
TABLE II

CHARACTERISTICS OF RADAR BRISBANE-AUSTRALIA

\begin{tabular}{l|c|c|c|c|c|c|c}
\hline Frequency & Band & PPI & Latitude & Longitude & Altitude & PRF & Range \\
\hline $2,753 \mathrm{GHz}$ & $\mathrm{S}$ & 10 & $-27,669$ & 152,862 & 168 & 999 & $150 \mathrm{Km}$ \\
\hline
\end{tabular}

Fractional uncertainty for each of the variables involved in the radar equation was assessed as Type-B uncertainty, also considering the information provided by suppliers (manufacturers).

In the cases where suppliers provide the maximum error for a particular variable, the following expression serves to compute the fractional uncertainty of a triangular distribution:

$$
\frac{u(x)}{x}=\frac{\Delta \mathrm{X}}{\sqrt{6}}
$$

Similarly, for a normal distribution we can use:

$$
\frac{u(x)}{x}=\frac{\Delta \mathrm{X}}{3}
$$

On the other hand, if suppliers provide relative uncertainty in $\mathrm{dB}$, it becomes necessary to calculate the corresponding fractional uncertainty as follows [9]:

$$
\frac{u(x)}{x}=10^{\frac{u(x) d B}{10}}-1
$$

\section{A. Antenna}

TABLE III

ANTENNA UNCERTAINTY

\begin{tabular}{c|c|c|c|c|c|c}
\hline \hline Parameter & Value & $\begin{array}{c}\text { Error } \\
\pm \Delta \mathrm{X}\end{array}$ & $\begin{array}{c}\text { Relative } \\
\text { uncertainty } \\
\mathrm{u}(\mathrm{x})\end{array}$ & $\begin{array}{c}\text { Linear } \\
\text { scale } \\
\text { Error } \\
\pm \Delta \mathrm{X}\end{array}$ & $\begin{array}{c}\text { Fractional } \\
\text { uncertainty } \\
\text { Triangular } \\
\frac{u(x)}{x}\end{array}$ & $\begin{array}{c}\text { Fractional } \\
\text { uncertainty } \\
\text { Normal } \\
\frac{u(x)}{x}\end{array}$ \\
\hline Gain & $45 \mathrm{~dB}$ & ----- & $0.5 \mathrm{~dB}$ & ---- & 0.122 & 0.122 \\
\hline Angle & $1^{\circ}$ & $0.03^{\circ}$ & & 0.03 & 0.012 & 0.01 \\
\hline \hline
\end{tabular}

\section{B. Radome Associated Loss}

Regarding the information provided by antenna suppliers, the standard uncertainty due to radome loss is about $0.02 \mathrm{~dB}$.

TABLE IV RADOME LOSS

\begin{tabular}{c|c|c|c|c}
\hline \hline Parameter & Value & $\begin{array}{c}\text { Relative } \\
\text { Uncertainty } \\
\mathrm{u}(\mathrm{x})\end{array}$ & Linear scale & $\begin{array}{c}\text { Fractional } \\
\text { uncertainty } \\
\frac{u(x)}{x}\end{array}$ \\
\hline Radome Loss & $0.5 \mathrm{~dB}$ & $0.02 \mathrm{~dB}$ & 0.0046 & 0.0046 \\
\hline
\end{tabular}

C. Pulse length $\tau$

TABLE V PULSE-LENGTH UNCERTAINTY T.

\begin{tabular}{c|c|c|c|c}
\hline Parameter & Value & Error $\pm \Delta \mathrm{X}$ & $\begin{array}{c}\text { Fractional } \\
\text { uncertainty } \\
\text { Triangular } \\
\frac{u(x)}{x}\end{array}$ & $\begin{array}{c}\text { Fractional } \\
\text { uncertainty } \\
- \text { Normal } \\
\frac{u(x)}{x}\end{array}$ \\
\hline Pulse length $\tau$ & $0,0002185979 \mathrm{seg}$ & $0.000020 \mathrm{seg}$ & 0.037 & 0.03049 \\
\hline \hline
\end{tabular}




\section{Frequency}

TABLE VI

FREQUENCY UNCERTAINTY

\begin{tabular}{c|c|c|c|c|c}
\hline Parameter & Value & Error $\pm \Delta \mathrm{X}$ & $\begin{array}{c}\text { Linear } \\
\text { scale }\end{array}$ & $\begin{array}{c}\text { Fractional } \\
\text { uncertainty } \\
\text { Triangular } \\
\frac{u(x)}{x}\end{array}$ & $\begin{array}{c}\text { Fractional } \\
\text { uncertainty } \\
\text { Normal } \\
\frac{u(x)}{x}\end{array}$ \\
\hline Frequency & $2,753 \mathrm{GHz}$ & $0.004 \mathrm{GHz}$ & 0.004 & 0.0016 & 0.0013 \\
\hline \hline
\end{tabular}

E. Transmitted power

TABLE VII

TRANSMITTED-POWER UNCERTAINTY

\begin{tabular}{c|c|c|c}
\hline \hline Parameter & Value & $\begin{array}{c}\text { Relative uncertainty } \\
u(x)\end{array}$ & $\begin{array}{c}\text { Fractional uncertainty } \\
\frac{u(x)}{x}\end{array}$ \\
\hline Power & $250 \mathrm{KW}$ & $25 \mathrm{KW}$ & 0.10 \\
\hline \hline
\end{tabular}

F. Receiving-filter loss

TABLE VIII

RECEIVING-FILTER LOSS (UNCERTAINTY)

\begin{tabular}{c|c|c|c|c}
\hline \hline Parameter & Value & $\begin{array}{c}\text { Relative } \\
\text { uncertainty } \\
u(x)\end{array}$ & $\begin{array}{c}\text { Linear } \\
\text { scale }\end{array}$ & $\begin{array}{c}\text { Fractional } \\
\text { uncertainty } \\
\frac{u(x)}{x}\end{array}$ \\
\hline $\begin{array}{c}\text { Receiving- } \\
\text { filter loss }\end{array}$ & $0.02 \mathrm{~dB}$ & 0.0471 & 0.0471 \\
\hline \hline
\end{tabular}

Finally, by using equation 21, it is possible to calculate the fractional uncertainty associated to that radar constant, as shown in Table IX:

TABLE IX

RADAR-CONSTANT UNCERTAINTY

\begin{tabular}{c|c|c}
\hline \multicolumn{2}{c}{$\left[\frac{u_{c}\left(C_{R}\right)^{2}}{C_{R}}\right]^{2}=\left[\frac{2 u\left(C_{A N T}\right)_{A N T}^{2}}{C_{A N}^{2}+\left[\frac{u\left(\mathrm{P}_{\mathrm{tx}}\right)}{P_{t x}}\right]^{2}+\left[\frac{\left.L_{R D}\right)^{2}}{L_{M F}}\right]^{2}+\left[\frac{u(\tau)}{\tau}\right]^{2}+\left[\frac{2 u(\mathrm{f})}{f}\right]^{2}}\right.$} \\
\hline Fractional Uncertainty & \multicolumn{1}{c}{ Triangular value } & Normal value \\
\hline$\frac{u\left(C_{A N T}\right)}{C_{A N T}}$ & 0.134 & 0.132 \\
\hline$\frac{u\left(L_{R D}\right)}{L_{R D}}$ & 0.0046 & 0.0046 \\
\hline$\frac{u(\tau)}{\tau}$ & 0.037 & 0.030 \\
\hline$\frac{2 u(\mathrm{f})}{f}$ & 0.0016 & 0.0013 \\
\hline$\frac{u\left(\mathrm{P}_{\mathrm{tx}}\right)}{P_{t x}}$ & 0.10 & 0.10 \\
\hline$\frac{u\left(\mathrm{~L}_{\mathrm{MF}}\right)}{L_{M F}}$ & 0.0471 & 0.0471 \\
\hline$\frac{u_{c}\left(C_{R}\right)}{C_{R}}$ & 0.2924 & 0.2879 \\
\hline \hline
\end{tabular}

Using one example of the received power, namely $-85 \mathrm{dBm}$ at a distance of 10 kilometers, the following uncertainty calculations were obtained in terms of reflectivity:

G. Received power

TABLE X

RECEIVED-POWER UNCERTAINTY

\begin{tabular}{c|c|c|c}
\hline Parameter & Value & $\begin{array}{c}\text { Relative } \\
\text { uncertainty } \\
u(x)\end{array}$ & $\begin{array}{c}\text { Fractional } \\
\text { uncertainty } \\
\frac{u(x)}{x}\end{array}$ \\
\hline Potencia & $-85 \mathrm{dBm}$ & $0.7 \mathrm{~dB}$ & 0.17 \\
\hline
\end{tabular}

\section{H. Distance to target}

TABLE XI

RADAR-DISTANCE UNCERTAINTY

\begin{tabular}{c|c|c|c}
\hline Parameter & Value & $\begin{array}{c}\text { Relative } \\
\text { uncertainty } \\
u(x)\end{array}$ & $\begin{array}{c}\text { Fractional uncertainty } \\
\frac{u(x)}{x}\end{array}$ \\
\hline Distance & $10 \mathrm{Km}$ & $0.1 \mathrm{~km}$ & 0.10 \\
\hline
\end{tabular}

\section{Attenuation loss}

The reciprocal value of $L_{p}$ ranges from 0 to 1 , and, when having ideal conditions, this factor is assumed to be equal to $1 ; L_{p}$ is defined as follows:

$$
L_{p}\left(r_{o}\right)=e^{\int_{0}^{r_{o}}\left(k_{g}+k_{n}+k_{p}\right) d r}
$$

Where $k_{g}\left(\frac{\mathrm{dB}}{\mathrm{Km}}\right)$ is the attenuation coefficient of gases, $\mathrm{k}_{\mathrm{n}}(\mathrm{dB} / \mathrm{Km})$ corresponds to the absorption coefficient of clouds, and $\mathrm{k}_{\mathrm{p}}\left(\frac{\mathrm{dB}}{\mathrm{Km}}\right)$ is the attenuation coefficient of rain. All these coefficients take approximate values intended for an S-band radar [10]:

Specifically, for an S-band radar, attenuation of gases is negligible, thus, $k_{g}=0 \mathrm{~dB} / \mathrm{Km}, k_{n}=0.004 \mathrm{~dB} / \mathrm{Km}$, and $k_{p}=0.004 \mathrm{~dB} / \mathrm{Km}$, for precipitations of about $12 \mathrm{~mm} / \mathrm{h}$ [11] .According to these values, we obtain $\mathrm{L}_{\mathrm{p}}=1.072$ together with its reciprocal $L_{p}{ }^{-1}=0.9323$.

TABLE XII

UNCERTAINTY DUE TO ATTENUATION

\begin{tabular}{c|c|c|c}
\hline \hline Parameter & $\begin{array}{c}\text { Value } \\
L_{p}^{-1}\end{array}$ & $\begin{array}{c}\text { Relative } \\
\text { Uncertainty } \\
u(x)\end{array}$ & $\begin{array}{c}\text { Fractional } \\
\text { Uncertainty } \\
\frac{u(x)}{x}\end{array}$ \\
\hline Attenuation loss & 0.9323 & 0.01 & 0.010 \\
\hline \hline
\end{tabular}

In order to calculate the fractional uncertainty of reflectivity, we use equation (19). These results can be observed in Table XIII.

TABLE XIII REFLECTIVITY UNCERTAINTY

\begin{tabular}{c|c|c}
\hline \multicolumn{2}{c}{$\left[\frac{u_{c}(z)^{2}}{Z}\right]^{2}=\left[\frac{u\left(C_{R}\right)}{C_{R}}\right]^{2}+\left[2 \frac{u\left(L_{p}\right)}{L_{p}}+2 \frac{u(r)}{r}\right]^{2}+\left[\frac{u\left(P_{r}\right)}{P_{r}}\right]^{2}$} \\
\hline Fractional Uncertainty & Triangular Value & Normal Value \\
\hline$\frac{u\left(C_{R}\right)}{C_{R}}$ & 0.2924 & 0.2879 \\
\hline$\frac{u\left(L_{p}\right)}{L_{p}}$ & 0.010 & 0.010 \\
\hline$\frac{u(r)}{r}$ & 0.10 & 0.10 \\
\hline$\frac{u\left(P_{r}\right)}{P_{r}}$ & 0.17 & 0.17 \\
\hline$\frac{u_{c}(z)}{Z}$ & 0.4034 & 0.4002 \\
\hline
\end{tabular}

By using these data and also considering a sample reflectivity measurement of $40 \mathrm{~dB}\left(10000 \mathrm{~mm}^{\wedge} 6 / \mathrm{m}^{\wedge} 3\right)$, the following values are obtained: 
Linear scale:

$$
z=10000 \pm 4002 \frac{\mathrm{mm}^{6}}{\mathrm{~m}^{3}}
$$

Logarithmic scale:

$$
Z=40 d B \pm 1.5 d B
$$

Provided there is considerable reflectivity uncertainty, it is worth noting that all this uncertainty propagates over the final precipitation estimation.

For the computation of precipitation uncertainty, the most common $\mathrm{Z}-\mathrm{R}$ empirical relation is adopted [12]

$$
Z=a R^{b}
$$

Solving for R, we obtain:

$$
R=\left(\frac{Z}{a}\right)^{\frac{1}{b}}
$$

Teni Using equation (25), the following expression is obtained to compute the precipitation uncertainty:

$$
u_{c}^{2}(R)=\left[\frac{\partial R}{\partial Z}\right]^{2} u^{2}(Z)+\left[\frac{\partial R}{\partial a}\right]^{2} u^{2}(a)+\left[\frac{\partial R}{\partial b}\right]^{2} u^{2}(b)
$$

The following table shows the results obtained from the partial derivatives:

TABLE XIV

PARTIAL DERIVATIVES FOR THE PRECIPITATION UNCERTAINTY MODEL

\begin{tabular}{c|c|c|c}
\hline \multicolumn{3}{c}{$R=\left(\frac{Z}{a}\right)^{\frac{1}{b}}=\sqrt[b]{\frac{Z}{a}}$} \\
\hline$C_{1}$ & $\frac{\partial R}{\partial Z}$ & $\frac{1}{b Z} \sqrt[b]{\frac{Z}{a}}$ & $\frac{R}{b Z}$ \\
\hline$\overline{C_{2}}$ & $\frac{\partial R}{\partial a}$ & $-\frac{1}{b a} \sqrt[b]{\frac{Z}{a}}$ & $\frac{R}{-\frac{R}{b a}}$ \\
\hline$C_{3}$ & $\frac{\partial R}{\partial b}$ & $-\frac{1}{b^{2}} \sqrt[b]{\frac{Z}{a}} \ln \left[\frac{Z}{a}\right]$ & $-\frac{R}{b^{2}} \ln \left[\frac{Z}{a}\right]$ \\
\hline
\end{tabular}

By substituting the partial derivatives we obtain:

$$
\begin{gathered}
u_{c}^{2}(R)=\left[\frac{R}{b Z}\right]^{2} u^{2}(Z)+\left[\frac{R}{b a}\right]^{2} u^{2}(a)+\left[\frac{R}{b^{2}} \ln \left[\frac{Z}{a}\right]\right]^{2} u^{2}(b) \\
{\left[\frac{u_{c}(R)}{R}\right]^{2}=\left[\frac{u^{2}(Z)}{b Z}\right]^{2}+\left[\frac{u^{2}(a)}{b a}\right]^{2}+\left[\frac{u^{2}(b)}{b^{2}} \ln \left[\frac{Z}{a}\right]\right]}
\end{gathered}
$$

Finally, the following expression can be obtained to find the fractional uncertainty of precipitation using the Z-R relation:

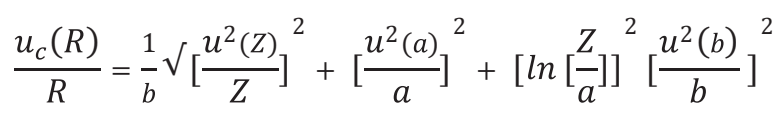

Based on the previous expression, it is possible to calculate the Type-A fractional uncertainty for constants " $a$ " and " $b$ " from the data provided in the literature regarding various Z-R relations.
TABLE XV

A SUMMARY OF DIFFERENT VALUES FOR “A” AND “B” REGARDING Z-R RELATIONS [13], [14].

\begin{tabular}{c|c|c|c|c|c}
\hline $\mathrm{a}$ & $\mathrm{b}$ & $\mathrm{a}$ & $\mathrm{b}$ & $\mathrm{a}$ & $\mathrm{b}$ \\
\hline 200 & 1,6 & 300 & 1,4 & 124 & 1,64 \\
\hline 300 & 1,35 & 21 & 1,71 & 667 & 1,33 \\
\hline 176,5 & 1,29 & 486 & 1,37 & 500 & 1,5 \\
\hline 215,9 & 1,35 & 300 & 1,5 & 450 & 1,46 \\
\hline 171,9 & 1,19 & 250 & 1,2 & 200 & 1,6 \\
\hline 172,8 & 1,33 & 130 & 2 & 800 & 1,6 \\
\hline 371 & 1,24 & 75 & 2 & 348 & 1,81 \\
\hline 162 & 1,48 & 140 & 1,5 & 134 & 1,55 \\
\hline 167,8 & 1,26 & 250 & 1,2 & 162 & 1,48 \\
\hline 65,5 & 1,69 & 436 & 1,43 & 371 & 1,24 \\
\hline
\end{tabular}

To calculate the Type-A standard uncertainty of "a" and "b" equation (3) was used, yielding the following values:

TABLE XVI

PRECIPITATION UNCERTAINTY

\begin{tabular}{c|c|c}
\hline Fractional Uncertainty & Triangular value & Normal value \\
\hline$\frac{u_{c}(z)}{Z}$ & 0,4034 & 0,4002 \\
\hline$\frac{u^{2}(a)}{a}$ & 0,1197 & 0,1197 \\
\hline$\frac{u^{2}(b)}{b}$ & 0,02624 & 0,02624 \\
\hline$\frac{u_{c}(R)}{R}$ & 0.2920 & 0.290 \\
\hline
\end{tabular}

Specifically, when reflectivity is equal to $10000 \frac{\mathrm{mm}^{6}}{\mathrm{~m}^{3}}, \mathrm{a}=271.58$, and $b=1.476$, the following uncertainty values can be calculated for precipitation $(\mathrm{R})$ :

$$
\begin{aligned}
& R=\left(\frac{10000}{271.58}\right)^{\frac{1}{1.476}} \\
& R=11.49 \mathrm{~mm} \pm 29 \% \\
& R=11.49 \pm 3.33 \mathrm{~mm}
\end{aligned}
$$

\section{CONCLUSIONS}

The antenna represents the element that most contributes to introducing uncertainty in the radar's constant; therefore, antennas play a crucial role in calibration processes. Additionally, a lot of effort should go into estimating the actual antenna gain and beam-width angles due to the nature of these variables as well as to the external uncertainty sources involved.

Although the fractional uncertainty associated to the radar's constant is $40 \%$, when propagating towards the Marshall \& Palmer Z-R relation used herein (for precipitation estimation), its value decreases down to $29 \%$. The previous result follows from the very nature of the resulting expression for estimating precipitation in the Z-R relation.

The present work does not deal with all the possible uncertainty sources that affect quantitative precipitation estimation using radars. Specifically, aspects such as the space-time variation of the drop size distribution (dsd) would undoubtedly contribute to having greater 
uncertainty in the final results of similar studies. Furthermore, other uncertainty sources were also omitted, e.g. the uncertainty related to the non-linearity of transmitters and receivers, the partial beam filling, evaporation and condensation. These other uncertainty sources represent an interesting challenge for future research on precipitation estimation uncertainty using weather radars.

\section{REFERENCES}

[1] BIPM, IEC, IFCC, ILAC, ISO, IUPAC, IUPAP, and OIML, "Evaluation of measurement- Guide to the expression of uncertainty in measurement (JCGM 100:2008)," $1^{\text {a }}$ ed, Sep, 2008. [Online]. Available: http://www. bipm.org/utils/common/documents/jcgm/JCGM_100_2008_E.pdf

[2] G. Villarini, F. Serinaldi, F. Witold, and A. Krajewski, "Modeling radarrainfall estimation uncertainties using parametric and non-parametric approaches," Advances in Water Resources, vol. 31, pp. 1675-1685, April, 2008.

[3] B. Taylor, Barry N. Chris, and E. Kuyatt. "Guidelines for Evaluating and Expressing the Uncertainty of NIST Measurement Results. NIST Technical Note 1297," National Institute of Standards and TechnologyPhysics Laboratory, Sep, 1994. [Online]. Available: http://physics.nist. gov/Pubs/guidelines/TN1297/tn1297s.pdf

[4] I. Zawadski, "Factors affecting the precisión of radar measurements of rain," Preprints 22 nd Conf. of Radar Meteorology, Zurich, Switzerland, pp. 251-256, Sept, 1984.

[5] P. Jones, "The radar equation in meteorology," Quarterly Journal of the Royal Meteorological Society, pp. 485-495, Oct, 1962.

[6] R. Doviak, and D. Zrnic, "Matched filter criteria and range weighting for weather radar", IEEE Transactions Aerospace Electronic System, vol. AES 14, no. 6, pp. 925-929, Nov, 1978.

[7] R. Doviak and D. Zrnic, "Receiver Bandwidth effect on Reflectivity and Doppler Velocity estimates," Journal of Applied Meteorology, pp: 69-76, Jan, 1979.

[8] F. Gekat, M. Hille, H. Niese, and M. Pool, "Accuracy of the engineering calibration of weather radars," Geoscience and Remote Sensing Symposium (IGARSS), IEEE International, pp. 1096-1099, Nov, 2010.

[9] R. E. Rinehart, "Radar for Meteorologist", Nevada, Rinehart Publ, 6 a ed, pp. 365-380, Dec, 2006.

[10] R. Wexler, and D. Atlas, " Radar reflectivity and attenuation of rain," Journal of Applied Meteorology, vol. 2, pp. 276-280. Oct, 1963.

[11] K. L. Gunn, and T.W. East, "The microwave properties of precipitation particles," Quarterly Journal of the Royal Meteorological Society, pp.522-545, Jul,1954.

[12] J. Marshall and W. Palmer, "The distribution of raindrops with size," Journal of Meteorology, McGill University, Montreal, vol 5, pp. 165-16, Oct, 1948.

[13] L.J. Battan, " Radar observation of the atmosphere," University of Chicago Press, pp. 323-324, 1973.

[14] J. Copete, "Herramientas matemáticas e informáticas aplicables en la calibración y funcionamiento de un radar meteorológico concebido para el estudio del drenaje urbano de la ciudad de Bogotá," Universidad Nacional de Colombia, Maestría en ingeniería y recursos Hidráulicos, pp 56-58, Jul, 2009.

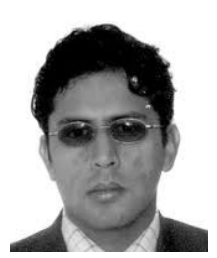

A. Ernesto Gómez Vargas was born in Duitama, Colombia, in 1978. He received the B.S. degree in electronic engineering from Distrital Francisco Jose de Caldas University, Bogota, Colombia, in 2000 and the M.S. degree in telecommunications from Distrital Francisco Jose de Caldas University, Bogota, Colombia, in 2004 and the Ph.D. degree in engineering from Javeriana University, Bogota, Colombia in 2015. From 2001, he has been an Assistant Professor with the Engineering Department, University of the District, Bogota, Colombia. He is the author of one book, and some articles. His research interests include weather radars, satellites and tele-communications.

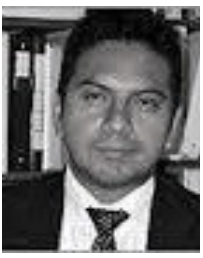

B. Nelson Obregón was born in Cúcuta City, Colombia, in 1967. He received the B.S. in Civil Engineering from Francisco de Paula Santander University of, Cúcuta, Colombia, in 1991; the M.S. degrees in Civil Engineering from the Andes University, Bogotá, Colombia, in 1998 and the $\mathrm{Ph} . \mathrm{D}$. degree in Hydrological Sciences from University of California, Davis, USA in 1998. From 1999, he has been an Full Professor with the Civil Engineering Department at Javeriana University, Bogotá, Colombia. From 2006 to date he also has been the head of Geophysical Institute at the same University. He is the author of several chapter books and more than 50 articles. His research interests include non-linear dynamics and chaos theory, hydroinformatics, complexity, artificial inteligence in geosciences and engineering.

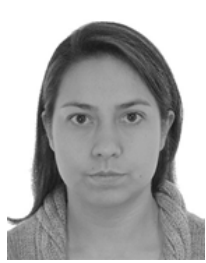

Lindsay Álvarez Pomar was born in Florencia, Colombia, in 1978. She received the B.S. degree in industrial engineering from Distrital Francisco Jose de Caldas University, Bogota, Colombia, in 2000 and the M.S. degree in industrial engineering from Andes University, Bogota, Colombia, in 2004. She is currently pursuing the Ph.D. degree in engineering at Distrital Francisco Jose de Caldas University, Bogota, Colombia. From 2001, she has been an Assistant Professor with the Industrial Engineering Department, University of the District, Bogota, Colombia. She is the author of three books, and some articles. Her research interests include simulation, industrial dynamics and stochastic processes. Mrs. Alvarez awards the first Latin American simulation competition in 2008 . 